\title{
METAMORPHOSIS OF FILARIA IN THE BODY OF THE MOSQUITO (CULEX PIPIENS).
}

\author{
MaRio. G. LEBREDo, \\ Vice-Director of "Las Animas" Hospital, Havana, Cuba.
}

\section{INTRODUCIION.}

The names of Demarquay, Lewis, Manson, Cobbold, Low and James may be said to epitomize the history of filaria. They may be divided into two groups; one representing the fortunate discoverers of these curious nematodes, and the other, including Manson, Low and James, the students of the transformations of the parasite in the mosquito.

If we consider that filaria, as demonstrated by Manson, is transmitted by the mosquito, we are disposed to conclude that the prevention of its propagation must be an easy matter; and, theoretically, it is so, provided we can secure a strict isolation of infected patients from the bite of mosquitoes.

There are still some points that require further elucidation, such as the mode of transmission from the mosquito to man, but, from the sanitary viewpoint, it is sufficient to know that the evolution of the filaria embryo occurs exclusively in the mosquito. This knowledge has not been applied in sanitary practice.

My own investigations have concerned themselves with the evolution of the parasite in the body of the mosquito, and with the determination of how, where, and when the worm leaves the body of the insect. My aim has been to include the whole process, and to leave no doubt as to the inoculation of filaria by mosquitoes in the human subject.

\section{SOURCE OF MATERIAL.}

The case utilized for my experiments was one of moderate infection. He was sent to me by Dr. Enrique Nuñez and he subjected himself patiently to many annoying procedures.

The clinical history is briefly as follows:

He had always enjoyed good health, except that he was operated on in June 1903 , by Dr. Nuñez. for a left inguinal hernia.

He remembers to have had, since he was eight years old, a slight enlargement of the glands in the right groin. This increased very gradually, extending downwards into the thigh. 
Recently the diagnosis of adenolymphocele was made, and the tumor was totally extirpated in January, 1904.

In July, an abscess, about the size of a hen's egg, formed in the left thigh. This was opened, and healed in about nine days. An examination of the blood made at this time revealed a considerable number of filaria embryos.

On the day of admission in "Las Animas" hospital, August 22, 1904, the blood was examined at 8:30 г. м. with negative results. On the following day, at 9:30 P. M. from two to three embryos were found in each preparation. A little later we could find from 10 to 12 in each preparation. We may look upon this as the average during the nights when mosquitoes have been applied.

\section{MOSQUTTOES, AND TECHNIQUE OF THEIR INFECTION WITH} FILARIAE.

Mosquitoes were applied to our patient on seven occasions, separated by the intervals of time necessary for the study of the infected insects. The conclusions of the present paper are founded on the first six applications. The seventh series is still under observation.

The facilities for this line of experiments at "Las Animas" Hospital are great, since we have rooms well screened with wire gauze, and assistants who have been well trained in mosquito work.*

Several kinds of mosquitoes have been used in these experiments, but the one in which we have been able to follow methodically the phases of development of filaria has been Culex pipiens. This particular species must be allowed to bite in condition of relative freedom, under a mosquito net. Our Anopheles (albipes), Stegomyia, and Culex consobrinus on the other hand, will bite readily in jars. The insects were made to bite at night, in accordance with the law of periodicity that causes the filaria nocturna to appear in the peripheral circulation only at night.

In order to obtain infection of the insects by the greatest number of embryos possible, it is advisable to have the patient go to bed, at least two hours before the mosquitoes are let loose under the net. Our failure to comply with this requirement caused a marked diminution of the infection in one of our series.

After remaining under the net all night with the patient, the insects were collected on the following morning.

* I have to thank Mr. E. Gros, the assistant in the laboratory, for valuable aid in carrying out these investigations. 
The hour of 12 P. M. was fixed upon as the time of beginning of the infection. By that time all the insects had surely bitten.

The mosquitoes employed were mostly specimens reared in captivity. Some insects were captured in the attendants' dormitories, where there was no filariasis. There can be no doubt, therefore, that the mosquitoes had not been previously infected.

A curious difference is noticeable between the insects proceeding from these two sources. The insects reared in our breeding jars are much less likely to bite than those captured in adult life. In the former class $I$ have had only two out of 50 insects bite on some occasions; whereas hungry veterans captured in adult life are always successful biters.

Of 11 insects of the species Culex consobrinus, that had bitten the patient, not one became infected.

Out of 19 stegomyias, only one showed some infection, in the shape of two filariae that were still alive on the fifth day, but poorly developed.

Out of 70 specimens of Culex pipiens only eight failed to become infected, and these had been applied under the unfavorable conditions previously mentioned.

Of the varieties experimented with, Culex pipiens is without doubt the transmitter of this infection.

As bases for the conclusions arrived at in this paper, I wish to mention the following facts:

1. The female alone is capable of sucking blood.

2. The object of the blood is to assist in ovulation.

3. The insect does not perish after laying her eggs.

4. The duration of life of Culex pipiens is generally about two or three months.

5. The insect may bite every four or five days. It digests its blood meal in three or four days.

6. The female mosquito, kept in captivity, feeds after its first blood meal, on several kinds of food. Some experimenters feed them on fruit. In our laboratory we use sugar. A female mosquito will feed on sugar before she has digested the blood. I have dissected insects whose true stomach still contained blood and whose accessory stomach, or esophageal diverticulum, was full of syrup. 


\section{HISTOLOGICAL TECHNIQUE.}

For studying the distribution of the filariae in the body of the mosquito, sections are best used.

On the other hand, if we wish to follow the cycle of development of the parasite, of its dimensions and structure, we must dissect the infected mosquitoes.

TECHNIQUE FOR THE PREPARATION OF SECTIONS.

1. The live mosquito is placed in absolute alcohol. In this manner the penetration necessary for fixation and dehydration is obtained. Duration 24 hours.

2. Removal of wings and legs in the same liquid, in a watch glass.

3. Equal parts of absolute alcohol and ether, 24 hours.

4. Weak solution of celloidin, 24 hours.

5. Thick celloidin, 24 hours.

6. Mounting on block.

7. Chloroform, 20 minutes.

8. Eighty per cent alcohol until ready to cut.

The sections were always stained in Böhmers hematoxylin, followed in some cases by eosin and in others by Van Gieson's solution.

\section{DISSECTION.}

The living insect is placed in a test-tube with a small amount of water, and is shaken until the wings become wet, and the insect floats on the water. The liquid is poured into a watch glass, where the legs and wings are pulled off by means of forceps.

The further dissection should be made in the following order:

Dissection of the abdomen upon a slide, constantly irrigating the mosquito with distilled water or weak salt solution. After dissection place under microscope and determine by medium power whether there are any filariae. The head and thorax are transferred to another slide. Here the chitin is broken near the neck, and the head is transferred to another slide. Each part is thus dissected separately, and we are able to determine the localization of the parasites. If the latter be well advanced in their development, they push their way out through the openings made in the chitinous covering at the time of dissection.

If we wish to preserve the specimens, the filariae, if they are large enough, are transferred from place to place upon the slide, and repeatedly washed, while we clean and remove all detritus of the body of the mosquito from the slide. We finally add a drop of mixture of water, glycerin, and formalin, and the preparation is covered and cemented. Such preparations may be studied with the immersion lens.

When the filariae are too small to be isolated, it is best to leave them in the midst of the detritus, and to stain them as follows :

1. Allow them to dry on the slide.

2. Fix with 95 per cent alcohol, 1 to 2 minutes.

3. Place in water a few minutes.

4. Bohmer's hematoxylin, 1,2 , or 3 minutes, according to the intensity of the stain desired.

5 . Wash in running water, 3 to 5 minutes.

6. Watery .01 per cent solution of eosin, $1 / 2$ to 1 minute. 
7. Wash in 95 per cent alcohol.

8. Dry with paper.

9. Oil of cloves, 2 to 3 minutes.

10. Xylol.

11. Balsam.

The above technique may appear complicated, but it may be carried through in 8 to 10 minutes. Without staining, the filariae, when they are too small or too few, may escape observation.

The dissection above described is not a careful dissection of the insect, but should be considered rather as a coarse fragmentation into sections, as follows: the stomach, intestine and ovaries in one piece; the thoracic muscle in four or more bundles; and the head and mouth parts. Care should be exercised not to lose a single fragment.

\section{METAMORPHOSIS OF FILARIA IN MOSQUITO.}

I shall describe now the several steps in the metamorphosis of the filaria in the mosquito. I shall touch upon some points that have been in dispute, but upon which my opinions are free from all bias, since my observations were made before I had a complete knowledge of the literature.

\section{CHANGES OF POSITION OF THE FILARIA WITHIN ITS HOST, AND THE DATES COUNTING FROM THE FIRST DAY OF INFECTION, THAT COR- RESPOND TO SUCH CHANGES.}

Histologically we may say that the filariae are found in one or the other of two structures, namely: in the stomach, together with the blood ingested from an infected patient; or in th $\rightarrow$ loose connective tissue, called the fat body.

Topographically we find that filaria embryos may occupy the following positions: (1) they are taken into the stomach where they remain during a variable number of hours; (2) they then pass to the thorax where they lie as in a nest, undergoing the principal morphological changes; and (3) they usually pass out through the head and labium, but sometimes the worm loses its way in the body and becomes lodged in the abdomen under the chitinous covering.

The transformations of the filaria may be considered as follows in my several series :

First and second series. - At the end of 36 hours all the embryos had left the eavity of the stomach, and had wandered into the thorax. At the end of 12 days they were found in the neck.

Third and fourth series.--In 32 to 40 hours the embryos had passed to the thorax. As an example of unusual precocity I may mention one instance in which an embryo had found its way to the thorax 13 hours after the ingestion of blood in the stomach.

In these series filariae reachod the neck 13 days and 15 hours, and the labium 15 days and 14 hours after infection (stained preparations similar to those of Low). On the 20th, 30th and 38th day filariae were still found in 
the labium. One of the insects of this series presented, 15 days and nine hours after infection, 10 filariae in the head, and only three were still in the thorax.

Fifth series. - As in the other series nearly all the embryos had already left the stomach at the end of the first 40 hours. I should mention, however, one mosquito in whose thorax 21 filariae were found, and whose stomach filled with liquid blood still contained eight worms three days and nine hours after infection.

In this series the worms were still found in the thorax after 15 days and 14 hours, and their metamorphosis was not quite completed. They reached the head at the end of 19 days and nine hours. On the 30 th and 38th day they were still visible under the microscope, moving in the labium of the living insect.

Sixth series.-In this series the filariae reached the head after a period of 22 days and eight hours.

To recapitulate: In the first four series the filariae reached the head in 15 to 16 days. In the fifth series some delay was noticed, since filariae were found in the stomach after three days and nine hours, and none were found in the head on the 17 th day. In the sixth series the worms reached the head and labium on the $22 \pi$ day.

This difference in the time of migration is due to the fact that the embryos do not migrate until they have completed certain phases of their cycle of development. We shall consider later on the cause of this delay of development in some cases.

\section{DIMENSIONS AND GENERAL CONFIGURATION OF THE EMBRYOS IN THE DIFFERENT STAGES OF DEVELOPMENT.}

The morphological changes were found to vary in the different series in the same manner as the migrations of the worm.

In the following table all the series are presented together, and the maximum time, that corresponds with the several changes, is given.

The dimensions of the embryo filaria in the human blood are: length, from 0.130 to $0.300 \mathrm{~mm}$; width, 0.007 to $0.011 \mathrm{~mm} .^{1}$

\section{Dimensions of thr Filaria Embryos.}

In the stomach of the mosquitoes a few Length width hours after ingestion - - - $-0.203-0.277 \mathrm{~mm} .0 .0055-0.0074 \mathrm{~mm}$.

In the body of the mosquitoes during $\} \quad\left\{\begin{array}{lll}0.240 \mathrm{~mm} . & - & -0.0121 \mathrm{~mm} \\ 0.208 \mathrm{~mm} . & - & 0.0111 \mathrm{~mm}\end{array}\right.$. the first three days - - - $\} \quad\left\{\begin{array}{lll}0.208 \mathrm{~mm} . & - & 0.0111 \mathrm{~mm} \text {. } \\ 0.203 \mathrm{~mm} . & - & 0.0148 \mathrm{~mm} \text {. }\end{array}\right.$

In the three following days up to the $\} \quad\{0.192 \mathrm{~mm} . \quad-0.0185 \mathrm{~mm}$. sixth day - _ _ - - $\{0.176 \mathrm{~mm}$ - $-0.0194 \mathrm{~mm}$.

In the three following days up to $\{0.264 \mathrm{~mm}$. $-0.037 \mathrm{~mm}$. tenth day - . - -

During the following days the filaria continued to grow until it reached its full development on the 12th day in the more rapid, and the $22 \mathrm{~d}$ in the slower series

1 Dr. M. Braun, Die Thierischen Parasiten d. Menschen, Warzburg, 1903, p. 265. 
A careful study of the above figures will show that the development of the worm occurs in two stages. During the first stage the embryo grows shorter until it measures $0.160 \mathrm{~mm}$, and then it grows longer, and attains the maximum of $1.504 \mathrm{~mm}$. The width, on the other hand, changes in the opposite direction : during the first stage, it increases from $0.012 \mathrm{~mm}$. to $0.018 \mathrm{~mm}$. Finally, both dimensions increase; the length, much more rapidly than the width.

With thess changes in size we have also morphological changes. The shape of the embryo in the human blood is slender and delicate. After the ecdysis in the stomach of the mosquito, and soon after the migration to the thorax, the embryos become plump and sausage-like. Towards the end of the cycle of development the worm grows gradually longer and, though much larger, assumes again its original shape.

\section{STRUCTURAL CHANGES DURING THE CYCLE OF DEVELOPMENT.}

Fig. 1, A, gives a good idea of the hyaline sheath, fitting closely around the body of the embryo, but extending considerably beyond its length.

The embryo presents a somewhat square rather than rounded head. The posterior extremity is quite pointed. About one-third or one-fourth the length of the worm from the anterior extremity there is a clear space, the $V$-shaped space described by Manson. There is much doubt as to the embryonic significance of this space. A similar one is also found towards the posterior extremity. These spaces are quite apparent in stained preparations. In fresh preparations they appear as highly refractive spots.

In living embryos there is a remarkable series of refractive points or granules extending, rosary-like, from the middle of the embryo to about onequarter from the posterior extremity. The whole rosary moves backwards and forwards, but never advances beyond the center of the embryo.

The examination with high powers reveals a distinct transverse striation of the body (Fig. 2).

The anterior extremity or mouth has been described in divers manners. The highly refractive character of this region gives rise to much uncertainty. I have not been able to form any positive opinions as to the charact $r$ of the structure. In one instance only was I convinced of the existence of an arrangement consisting of three prismatic teeth. These teeth had the appear. ance and color of the teeth found in the maxillae of mosquitoes and somewhat yellowish color, such as is found in chitinous structures. The observation, however, is too isolated to warrant any final conclusions. The mobility of the living worm renders the o'servation more difficult. In stained preparations we see simply a sheath filled with minute granules.

The posterior extremity becomes remarkably changed during the metamorphis. From being sharp and pointed, it becomes the widest part of the worm.

Upon leaving the stomach of the mosquito, the filaria leaves its sheath in the coagulated blood. This operation is called ecdysis.

The first change visible after arrival in the thoracic muscles is a slight narrowing of the body of the embryo near its posterior fifth or sixth. This progresses slowly, and, at the same time, there is a gradual invagination of the 
posterior sixth, into the anterior portion. The worm grows shorter and thicker (Fig. 3).

The invagination continues until there is only a small portion of the pointed extremity protruding, like a small appendix from the thickened and rounded extremity.

The anterior extremity is somewhat square-shaped. The mouth, at this time, constitutes a round opening leading into a funnel shaped cavity. At the narrow termination of the latter we find the beginning of the digestive tract. This is now perfectly visible, extending to the anus. It becomes, however, more prominent when the filaria begins to elongate.

During this stage of elongation, the cephalic extremity does not change; excepting that, in some instances, a small, stiletto-like body is seen projecting from the oral funnel. The posterior or appendicular extremity suffers a retrograde metamorphosis. Although the worm has lost its outer sheath in the process of ecdysis, it is still surrounded by an adhering hyaline membrane. This fine integument, first described by James, becomes quite visible when it swells by imbibition. The structures contained within this membrane, at the end of the appendix, are seen to disappear, leaving an empty portion at the point. This portion appears then in the shape of a very small triangular, hyaline cap, upon the rounded posterior extremity.

The intestine can now be seen consisting of three distinct portions: one extending $0.384 \mathrm{~mm}$. backward from the bottom of the oral funnel. Here we find a constriction which reminds us of the esophageal bulb. From this point the intestine continues, forming a slightly undulating line to the anus. The latter appears at varying distances from the tail end, as may be seen in the following table:

\begin{tabular}{c|c|r}
\hline $\begin{array}{c}\text { Length } \\
\text { of Filaria }\end{array}$ & Width of Same & $\begin{array}{r}\text { Distance from Anus } \\
\text { to Tail End }\end{array}$ \\
\hline $0.192 \mathrm{~mm}$. & $0.0185 \mathrm{~mm}$. & $0.021-0.0240 \mathrm{~mm}$. \\
$0.336 \mathrm{~mm}$. & $0.02593 \mathrm{~mm}$. \\
$0.640 \mathrm{~mm}$. & $0.0370 \mathrm{~mm}$. & $0.0144 \mathrm{~mm}$. \\
$1.344 \mathrm{~mm}$. & $0.0320 \mathrm{~mm}$. & $0.056-0.0690 \mathrm{~mm}$. \\
\hline
\end{tabular}

Apparently the distance of the anus from the cauda increases; but in relation to the increase in length of the worm the distance really diminishes.

The diameter of the anal opening is greatest when the filaria is shortest; it becomes narrower and filliform as the embryo reaches its complete development in the mosquito.

I have seen a peculiar viscid substance leaving the anus, and remaining adherent to the filaria for some time. The substance appears at times to be inclosed in transparent vesicles.

As the embryo reaches its maximum length, the final ehanges occur in the caudal end; namely, the development of the three lobes. These are at first very small, but become later quite prominent. Sometimes they give the impression of a hook formation; but they are rounded, and symmetrically arranged around the point. 
I desire to call attention to a peculiar structure observed by me in three of the embryos examined. It was observed in the stage just preceding the final development. I do not mention it as a regular stage in the growth of the worm because $I$ have only observed it in a few instances.

This peculiar structure presents itself at the caudal extremity. It appears in the shape of a tube which starting from the posterior end, extends forwards, bending somewhat, and protruding sidewise at a short distance from the anus, where it projects forming a small conical protuberance (Fig. 4). The whole tube can be seen through the transparent tissues, and presents the shape of a sleeve, the cuff of which forms the conical elevation. This peculiar structure is quite motile. By movements that may be described as erectile the sleevelike organ is seen to bend, causing a lateral swelling of the terminal portion of the body of the filaria. At the same time the small titlike protuberance moves nearer or further from the anus. In this protuberance we are able to observe that the organ in question is hollow.

In a filaria measuring $1.344 \mathrm{~mm}$. in length, and $0.032 \mathrm{~mm}$. in width the following measurements were made (Fig. 4):

Distance from the anus (a) to the caudal extremity (b) .................. $0.069 \mathrm{~mm}$. Distance from the anus (a) to the nearest edge of the protruding cone (c) ....... $0.009 \mathrm{~mm}$. Distance from the tail end (b) to the nearest edge of the protruding cone (c) $\ldots .0 .045 \mathrm{~mm}$.

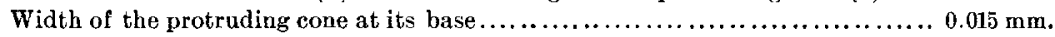

The titlike protuberance measures $0.0105 \mathrm{~mm}$. in height on the caudal side, and $0.006 \mathrm{~mm}$. on the side toward the anus.

During the erection the distension of the posterior portion always occurs on the side opposite the titlike projection. This expansion may extend to a distance from the tail end varying from $0.0525 \mathrm{~mm}$. to $0.069 \mathrm{~mm}$.

In measuring from the tail end the rounded extremity, not the appendix, was taken.

These measurements were made on living filariae anesthetized by adding a few drops of ether to the water.

I am not able to give any functional significance to this structure. I am sure that it is independent of the anus. Though no spicules are found in connection with the organ, the idea suggests itself that it may have some relation to the sexual organs.

The description of the steps in the metamorphosis of the embryo in the Culex pipiens may be condensed as follows:

1. The insect sucks the blood of a patient infected with filaria.

2. The embryo loses its sheath in the stomach of the mosquito, ecdysis.

3. Migration from the stomach to the thorax. This migration always takes place through the gastric wall, since both orifices of the gastric dilatation are completely closed when the stomach is full. The embryo leaves its sheath in the gastric contents or caught in the wall of the stomach, where it is left at the moment of exit. 
4. The embryo rests in the thorax, and goes through the following transformations:

(a) Narrowing and invagination of the tail.

(b) Invagination continues and the embryo grows shorter and wider.

(c) Widening and shortening continue, and the invaginated portion forms a hyaline appendix.

(d) Period of growth and formation of the three lobes.

The motility and non-motility of the embryo constitute biological features that are characteristic for the various phases of its development.

The active motility of the worm in the human circulation is further increased when the worm reaches the stomach of the mosquito. Upon reaching the thorax, however, all active motion ceases, and we only observe now and then very slight and sudden lateral movements. When the worm is nearing the maximnm of its growth we notice a slight increase in the lateral movements of the anterior extremity. As soon, however, as the embryo has completed its metamorphosis in the body of the mosquito, it recovers its motility, in order to accomplish its migration to the head. 'There the filaria awaits the opportunity to complete its cycle of development in the human host.

This latter motility is peculiar in that it enables the worm to push its way in the midst of soft tissues. It is a kind of motion that is quite distinct from that of the earlier stages when the animal has to move in liquids. I shall dwell further on upon these differences and shall use them in support of my views.

The worm, then, shows its activity in order to enter and to leave the thorax, and while in the latter it lies quiescent during its metamorphosis.

It happens sometimes, though rarely, that when the filaria reaches its maximum size, and starts on its way to the head, it may mistake the route, and wander towards the caudal extremity. The worm, however, will always keep in the fatty tissue, and close to the chitinous covering. These stray worms all proceed from the thorax. I have never met with a single embryo undergoing the process of metamorphosis in any other structure than the thoracic muscles. 
From three principal characters we may conclude that the filaria has completed its cycle of development in the mosquito, namely:

1. The arrival in the labium.

2. Complete development of the three lobes in the caudal extremity.

3. Active motility.

I desire now to consider the marked differences that were noticed, with respect to the time consumed in the cycle of development in my several series.

In his earlier investigations Manson saw embryos already in active motion seven days after infection. Bancroft ${ }^{1}$ says he never saw them before the 16th or 17th day, and in cold weather, even the 20th; James ${ }^{2}$ asserts that from 12 to 14 days are required for the complete metamorphosis, but he admits that, in localities where filaria abounds there may be species of mosquitoes that are peculiarly favorable to the development of the embryo. In this way he explains the seven days mentioned by Manson in his earlier work. He mentions the influence that climate and other factors may have upon these changes, and he reminds us of the fact that some of Manson's mosquitoes were kept in the incubator at temperatures ranging between $27^{\circ} \mathrm{C}$. and $29.5^{\circ} \mathrm{C}$.

We should not forget that the different results above mentioned were obtained in different countries. On the other hand I have also obtained the same variabiltiy in the results, though I was working in one locality, in the same laboratory, and with the same kind of mosquitoes.

The different results obtained by me, experimenting always with the same kind of mosquitoes, show that we cannot accept the suggestion of James to the effect that there may be species of mosquitoes that favor the development of the filaria.

Different species of mosquitoes may serve or not as hosts, but. if the insect is capable of acting as such, the time limit of the cycle extends within bounds that have not yet been fixed.

In my series of experiments the only variable point was the season of the year. The results obtained were the same for each

1 Tros, L. Bancroft, Jour, of Trop. Med., 1899, 2, p. 96.

2S. P. JAMES, Jour: of Trop. Med., 1900, 3, p. 46. 
series and the variations were noticeable only in the comparison of one series with another. The variations correspond, therefore, with the changes of temperature that prevailed at the time of experimenting with each series.

From the official bulletin of the meteorological station I have been able to obtain the mean temperature prevailing during the time of each series of observations, and I present the results in the following table.

\begin{tabular}{|c|c|c|c|}
\hline Series & Dates & $\begin{array}{l}\text { Complete devel- } \\
\text { opment of the } \\
\text { filaria in }\end{array}$ & $\begin{array}{l}\text { Mean temperature } \\
\text { for each period }\end{array}$ \\
\hline $\begin{array}{l}1,2,3, \ldots \ldots \\
4 \ldots \ldots \ldots \ldots \\
5 \ldots \ldots \ldots \ldots \\
6 \ldots \ldots \ldots \ldots\end{array}$ & $\left.\begin{array}{l}\text { August } 25 \text { to Sept. } 28 \\
\text { October } 12 \text { to Qct. } 27 \\
\text { Nov. } 9 \text { to Nov. } 26 \\
\text { December } 13 \text { to Jan. } 4\end{array}\right\}$ & $\begin{array}{r}15 \text { days } \\
19 \text { to } 23 \text { days }\end{array}$ & $\begin{array}{l}25.5^{\circ} \mathrm{C} . \\
\left.22.5^{\circ} \mathrm{C} .\right\}-21.8^{\circ} \mathrm{C} . \\
21.2^{\circ} \mathrm{C} . \mathrm{S}-2 .\end{array}$ \\
\hline
\end{tabular}

It is evident from the above table that the temperature is the chief factor in modifying the cycle of development. Heat is, then, the climatic condition that favors the development of the embryo.

The daily oscillations of the temperature were nearly the same during the time of the several series. The daily range was of $4.4^{\circ}, 4.5^{\circ}$ and $4.1^{\circ}$. The daily oscillations in Cuba, therefore, do not influence the growth of the worm.

I am preparing a series of experiments with mosquitoes kept in incubators at a uniform temperature, in order to fix the extremes of most favorable, and absolutely unfavorable temperatures.

THE ESCAPE OF THE WORM FROM THE INTERMEDIARY HOSTS.

The several kinds of living beings that may act as transmitters of disease, may be divided into two great groups. In one the infecting agent must go through a cycle of development within the host, without which the further transmission to another animal is impossible. In the other group the parasite, without undergoing any developmental changes is mechanically carried from one animal to another by an intermediary animal acting as an indifferent vehicle.

However, the act of infection by the first group of hosts may occur either actively or passively. In the first instance the con- 
veying animal, by its own act, inoculates the infecting agent; in the second, the intermediary host remains passive until the animal to be infected, takes up the infecting agent. An illustration of the first, or active mode of transmission, we have in the mosquito inoculating filaria by its own bite. We have in the hog, in whose flesh the cysticercus must wait passively until it is swallowed by man, an illustration of the second or passive mode of conveyance.

The filaria, leaving the body of man in the embryonal stage of its development must suffer a series of changes that can only take place in the body of the mosquito, before the worm can reach maturity, adult and sexual life, once more in the body of man; the mosquito (in my experiments Culex pipiens), is therefore the only transmitting agent.

When we take up the problem as to how this transmission is carried out, we find differences of opinion: some holding that the process is a passive one, and others that it is active. In the first instance it is maintained that the mature embryo passes from the body of the mosquito into drinking water, and is finally swallowed by man; in the second instance it is maintained that the mature embryo is directly inoculated by the mosquito. The former is the the old theory of Manson; the latter, the more modern of Low and James.

In attempting the solution of these problems, we must start with a thorough understanding of the facts presented in the earlier parts of this paper, specially with respect to the evidences of the final development of the embryo, its arrival in the labium, and the time that has passed since the mosquito used in the investigation became infected.

With all this information, I proceeded first to determine whether the filaria leaves spontaneously the body of the mosquito when the latter, still living, drops into the water, and care is taken that the integrity of the structure of the insect is preserved.

With this object in view I selected mosquitoes whose date of infection was well known, and in which the stage of development of the filariae was well established by the examination of other insects of the same series. 
The selected mosquitoes were placed in water in a watch-glass, and studied under the microscope. The results were always negative. The mosquitoes died without discharging a single worm. Upon dissection of these insects at the end of 24 or 28 hours, all the filariae within them were found dead, which is also the case when they are allowed to remain a few hours (up to 18) free in water.

That the integument of the insect was preserved in these cases was shown by the exit of large numbers of infusoria as soon as the chitinous covering was broken in the act of dissection.

This experience proves that, when a mosquito falls into the water if its cuticle be preserved, the flariae it may contain are unable to escape and perish by imbibition of water within a period of 24 hours.

My second series of experiments was intended to determine whether the filaria escapes at the time when the insect is feeding, or drinking, or when it lays its eggs. Be it understood that in these acts we exclude especially the sucking of blood.

Six mosquitoes, from among those most seriously infected, were placed in a separate jar on the sixth day of their infection. The water in the jar was examined every day.* The lumps of sugar hung up in little bags to feed the mosquitoes were also examined daily by dissolving them. The results of these experiments were always negative even up to the 38th day. At the end of this time while no filariae were found in the water, they were still to be found, living, and active, in the heads of the mosquitoes.

In other mosquitoes of the same series dissected at stated intervals, we were able to see that the filariae were fully developed, and had arrived in the labium on the 17 th day.

$\mathrm{My}$ second experiment proves, that the filaria does not pass from the living mosquito in the act of suction of water or sugar.

Whether the worm will pass into other kinds of food such as bananas, as has been suggested in connection with Filaria immitis by Grassi and Noé, is very doubtful. If the worm could pass into bananas and dates, it surely would do so in sugar.

*The daily examination of the water was made necessary in order to eliminate other organisms resembling the filariae that are likely to develop in water that has been standing some days. 
This same series of experiments, and other' observations, show that the filaria does not pass out at the time of laying the eggs.

There is one opportunity - the only one-for the filaria to find its way out of the mosquito into drinking water, and that is by accidental break in the integument; as may be shown experimentally in the act of dissection. But this must happen very rarely. Furthermore when the worms are set free in water they sink to the bottom and die by imbibition.

It appears then that only a series of accidents could bring the living filaria to the human stomach in the manner described.

The other hypothesis suggested to account for the transmission of the worm is that of a direct inoculation. There has been thus far no actual demonstration of the process.

Maitland argued that, "if it be true that the worm is introduced into the human body from the proboscis, we must suppose that the embryo must be always in position, ready to improve the opportunity given by the short time devoted to the act of suction." This is precisely what happens. The filaria is always ready.

The proof that the filaria remains day after day in the labium of the infected mosquito kept in captivity without a chance to repeat its feeding on blood, is found in the results obtained in sections by Low, and in the dissections made by James. These results suggested that the probable opportunity for escape is afforded in the act of drawing blood. The experiments here reported show that the migration does not occur during other acts of suction.

The experiments about to be described show how we may bring about at will the migration of the filaria, and permit the definite observation of the various steps in the process.

A mosquito is selected in which the filariae have reached their final stage of development, and are lodged in the labium. It is best to select an insect 28 to 30 days, or more, after infection. After removal of the wings and legs the insect is placed alive upon a slide and irrigated with a very weak salt solution. No cover-glass is employed. Under the microscope it is possible to see, through the transparent walls of the labium, the actively moving worms within that structure. 
If no pressure ${ }^{*}$ is used, these movements may be watched for hours without observing the escape of a single worm.

Usually all the setae are enclosed within the labium, thus preventing a clear view of the movements of the filaria. In order to obviate this difficulty I press very lightly with the side of a needle upon the base of the proboscis, and then with the point of a needle inserted between the setae and the labium, I pry them further apart. The filariae can now be distinctly seen actively moving and agitating the two tracheal tubes. The worms occupy usually the proximal third of the labium; sometimes they extend further out.

The slide is now placed near the flame of a Bunsen burner. This must be done very carefully in order to avoid fatal overheating to the insect and the parasite. Watching the preparation, an unusual activity in the movements of the filariae is now to be seen, and the cephalic end particularly becomes agitated as if seeking a point of exit. This cannot be found laterally because of the chitinous covering, and the filaria advances toward the anterior extremity of the labium. If the liquid is allowed to cool, the movements become slower and even cease altogether. Upon warming carefully again, and adding more tepid fluid, the movements are revived until the worms reach the point of the labium. If now the application of heat is stopped, we find that the worms appear to feel around with their cephalic extremity, but fail to break out.

I am. sure that there is no natural orifice at the terminal end of the labium, because if there were, the filaria, having reached this point, would find no obstacle to its exit. And, furthermore, a careful study of the extremity fails to discover any orifice.

I cannot agree with Grassi and Noé, quoted by Dutton, who

* Bancroft, in experimenting with Filaria immitis, cut off and mounted on a slide under a cover-glass the proboscis of an infected mosquito. By making slight pressure upon the cover-glass he forced the setae out from the labium. He observed under the microscope that upon increasing the pressure the actively moving worm protruded from the extremity of the labium. "Whether there be at this point," says Bancroft, "a natural opening, appears to be doubtful; but every time that this experiment was tried the worm made its exit from the point and never elsewhere."

However, when we compress the labium, a rupture of its anterior extremity occurs, and the exit of a considerable amount of the fatty contents takes place. The break occurs at the point mentioned because of the thickness and unyielding character of the tissues at. the end of the labellae. It is the break which offers a place of exit for the worms. 
suggest that "when the mosquito, in biting, pierces the skin, there is a rupture at the point, through strain, and the filaria escapes. They imply that the crowding of the filariae causes the strain and distension of the tissues.

In my experiment there is no skin to pierce. Furthermore, the previous extrusion of the setae has really diminished the strain at this point. Nor can there be any tension when we have only one or two filariae with plenty of room. Besides, we do not have the bend or elbow formed by the labium in the act of biting, and which might contribute to the strain suggested by Grassi and Noé, for the labium lies perfectly straight. Even when the number of the filariae is great there is no irregular rupture anywhere in the labium, as would result from over tension.

If the heat is kept up carefully and steadily we can see the embryo seeking the point, making pressure there, and finally perforating the cuticle at a certain point.

This perforation is made quite suddenly, for we see the cephalic end jump out, as it were; the rest of the worm following slowly by serpentine movements. As soon as it reaches the fluid the serpentine movements continue, but the worm ceases to be able to advance. This remarkable difference in the results obtained by the movement indicates that the soft tissues at this stage are the natural element of the worm, and that it is prepared to move on into the human tissues directly from the mosquito. In water, on the other hand, the filaria not only cannot live, but cannot even move from place to place.

In their exit the worms follow the regular order in which they occur in the labium and head. We have seen two worms making their exit at the same time (Fig. 6).

Occasionally, after two embryos have been started out, it has been necessary to warm the liquid again in order to bring other worms down from the head to the labium. Of course, after the first embryo has broken out, the others find their way out with greater readiness.

What temperature is best adapted to stimulate the passing out of the filaria, I am as yet unable to say, but I am inclined to think that the temperature should be rather high. A slight vapor 
should rise from the liquid, just enough to dim for a moment the lenses of the microscope.

Naturally enough the thought at once suggests itself of the importance of the heat in human blood as a factor in determining the exit of the filaria at the moment of biting.

\section{POINT OF EXIT OF FILARIA.}

Dutton describes a triangular space (seen in sections) near the point of the labium, and limited above by the chitinous band that forms the upper surface of the labium, and upon which the setae rest. He then adds: "This region appears to be the weak point in the chitinous exoskeleton of the labium; and it is probably at this point that the filaria escapes during the act of biting."

A careful study of the anatomy of the anterior extremity of the labium gives the following results:

The extremity of the labium presents an articulation indicated by a transverse groove (Fig. 5). The general appearance of this articulated end reminds us of the cloven hoof of certain animals. It is also divided symmetrically by a longitudinal groove, into two parts, called labellae. The separation, however, affects only the distal half of the end piece. In the proximal half the two sections are united by a membrane which, similar to an interdigital web, allows of free motion.

These two lateral bodies or labellae are cushioned with fatty tissue. The external (lateral) covering is chitinous and is rather thick. The internal (median) lining is a solt membrane covered with hairs.

I should mention that the whole inner lining of the labium is non-chitinous. It is a soft membrane made up of rounded cells. The filariae are lodged between this membrane and the outer cuticle.

When both labellae are united the longitudinal groove disappears and the whole structure appears as one piece.

Each labella has its own independent motion. When both segments are separated as far as the membrane (Fig. 5, B) will permit, we observe another complicated structure which protrudes pointlike between them, and forms with them a sort of tripod 
(Fig. 5, a). This central portion appears to be a sort of buttress or rib in the interlabellar membrane. It consists of a strong chitinous groove (Fig. 5, b) along the median line, and two (c) lateral, hairy portions, of semicircular shape, partially covering the groove. Between the two lateral portions there is seen an obtuse point, which represents the protruding posterior wall. The whole structure, excepting the groove, is formed of a delicate hairy tissue.

It is difficult to understand the object of this median portion. The hairy covering suggests a tactile function; but it is also possible that the object of these structures may be to bring about a close approximation of the epi- and hypopharynx.

All these well cushioned structures of the labellae are evidently intended to hold together and control the other portions of the proboscis when they are being introduced into the skin, in the act of biting. The control thus exercised, permits a certain amount of sliding motion at the same time that the setae are held rigidly together. The terminal portion also covers up completely the spot where the lancets penetrate.

When we watch with a strong lens the act of suction, we observe at once the backward bend of the labium with the penetrating setae forming the chord of the arch. The bend or elbow formed by the labium becomes the more acute, the greater the depth to which the penetrating elements are introduced. If the depth be small, at least one-third (terminal) of the length of the labium remains closed, forming a cylinder; but if the insect is obliged to push deeply in search of blood then the whole labium as far as the transverse furrow enters into the formation of the arch, and the penetrating elements of the proboscis are held together solely by the labellae which bend downward and backward at the articulation, and are placed in a position parallel with the penetrating setae.

At all events the extreme end of the labium is applied in very close contact with the skin surface at the point of penetration of the setae.

The filaria makes its exit always from one of the lateral points, that is, from the tip of one or the other labella. At these points, 
on the median aspect, the hairy integument is quite delicate, and readily perforated by the worm.

The place of perforation is, therefore, in close contact with the penetrating wound of the skin, and the setae. The latter are in constant motion (even movements of dilatation), and the best opportunity is offered for the successful inoculation of the parasite.

Having noted the extraordinary rapidity with which the filaria makes its exit, in one case five worms were seen to push their way out in less than one minute, it seemed important to determine the duration of the act of blood sucking. The mimimum of time was found to be a minute and a half, and the maximum four minutes. The mean time was two minutes; or more than enough for the mosquito to discharge its whole load of filariae into the wound.

Fully understanding all these phenomena, we cannot help but be impressed with the facility with which filarial infection is brought about, when the conditions are favorable.*

Fortunately the non-experimental infections frequently miscarry through failure of perhaps the most insignificant detail in the evolution of the parasite.

\section{EXPLANATION OF PLATES.}

(Original drawings by the author.)

Plate 1.

FIc. 1.-A. Embryo of filaria sanguinis hominis nocturna in human blood. B. Sheath of the embryo in the same preparation.

FIG. 2.-The same embryo of Fig. 1 under the immersion lens. The transverse striation of the cuticle is distinctly seen.

Plate 2.

FrG. 3.-1. Embryo in human blood-2 to 7. Stages of development with measurements of the embryo in the mosquito. The relative sizes represented in the figure are exact, and represent amplifications of 70 diameters. $(a),(b),(c)$, Process of invagination in the tail end of the embryo, seen as the worm passes from the sharp (1st) to the appendicular (2d) form.

Fia. $3^{1}$.-Anterior $(a)$ and posterior $(b)$ extremities of a fully developed embryo in the mosquito; $(c)$ anus.

* I have gathered mosquitoes from the room occupied by the patient, the subject of my experiments, and I have found filariae in the process of evolution. It would be interesting to examine systematically the blood of those individuals who have lived in contact with the patient. 
FIG. 4.-Peculiar formation seen in the caudal extremity of some embryos at the termination of the mosquito period of development; $(a)$ anus; (b) posterior extremity where the three characteristic lobes present themselves; (c) projecting tip forming the end of the sleeve, $(c) ;(d)$ portion of the sleeve that expands with the erectile movements of the same.

\section{Plate 3.}

Fig. 5.-A and B. Extremity of the labium. Anatomic study to show the point where the filariae make their exit. In Fig. A the lateral portions (labellae) are in contact; but in Fig. B they are separated to show the special structure $(a)$.

C. Special structure composed of (a), extreme blunt end; (b) chitinous groove; (c) lateral surfaces covered with fine hair.

Fia. 6.-Reproduction from nature of the exit of the filaria at the place of selection at the end of the labium. 
PLATE 1.

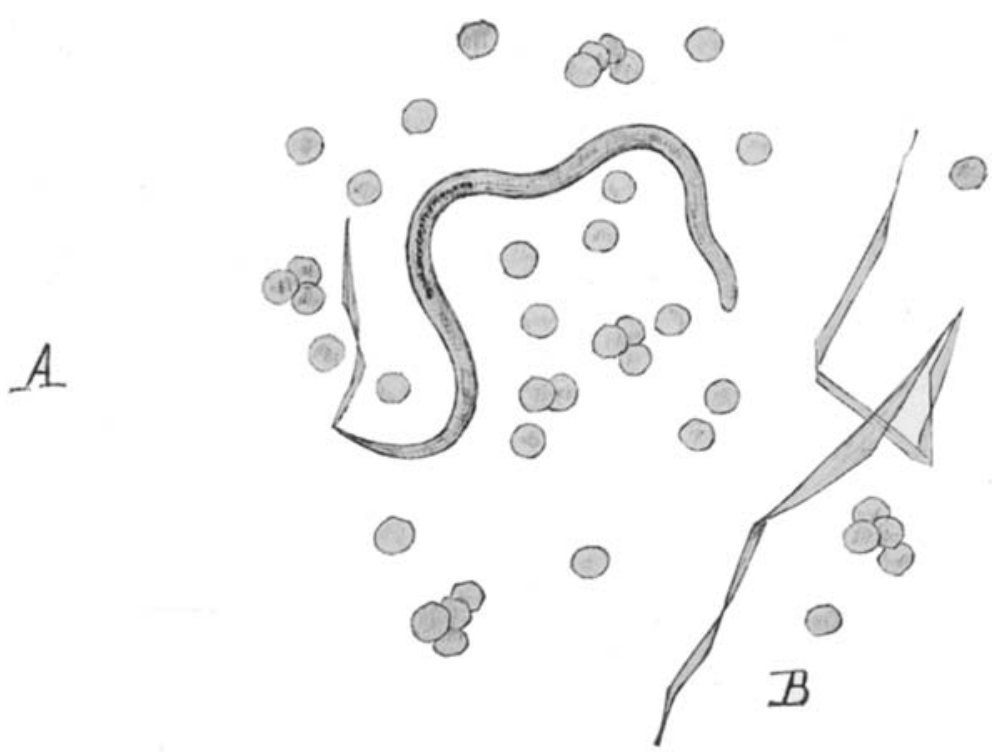

Frg. 1.

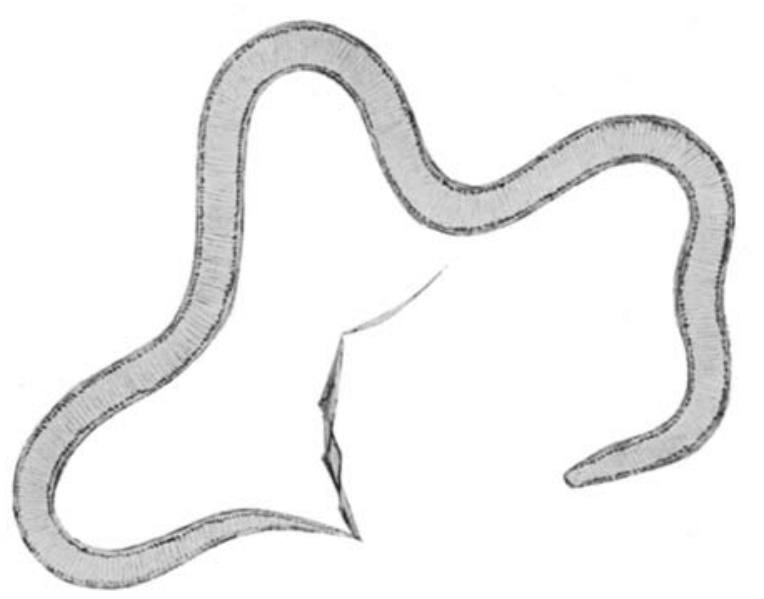

Fig. 2.

FIG. 2. 
PLATE 2.
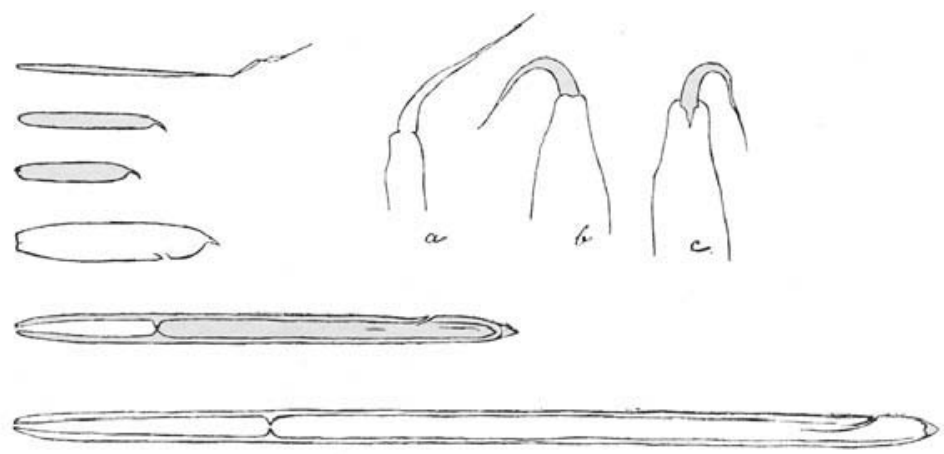

FIG. 3.

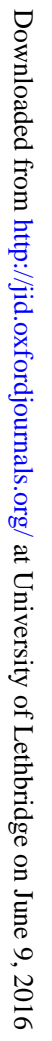

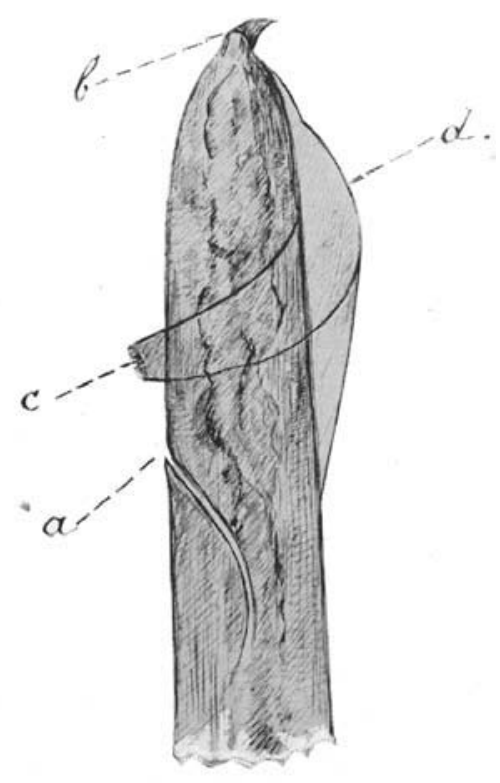

FIG. 4. 
PLATE 3.

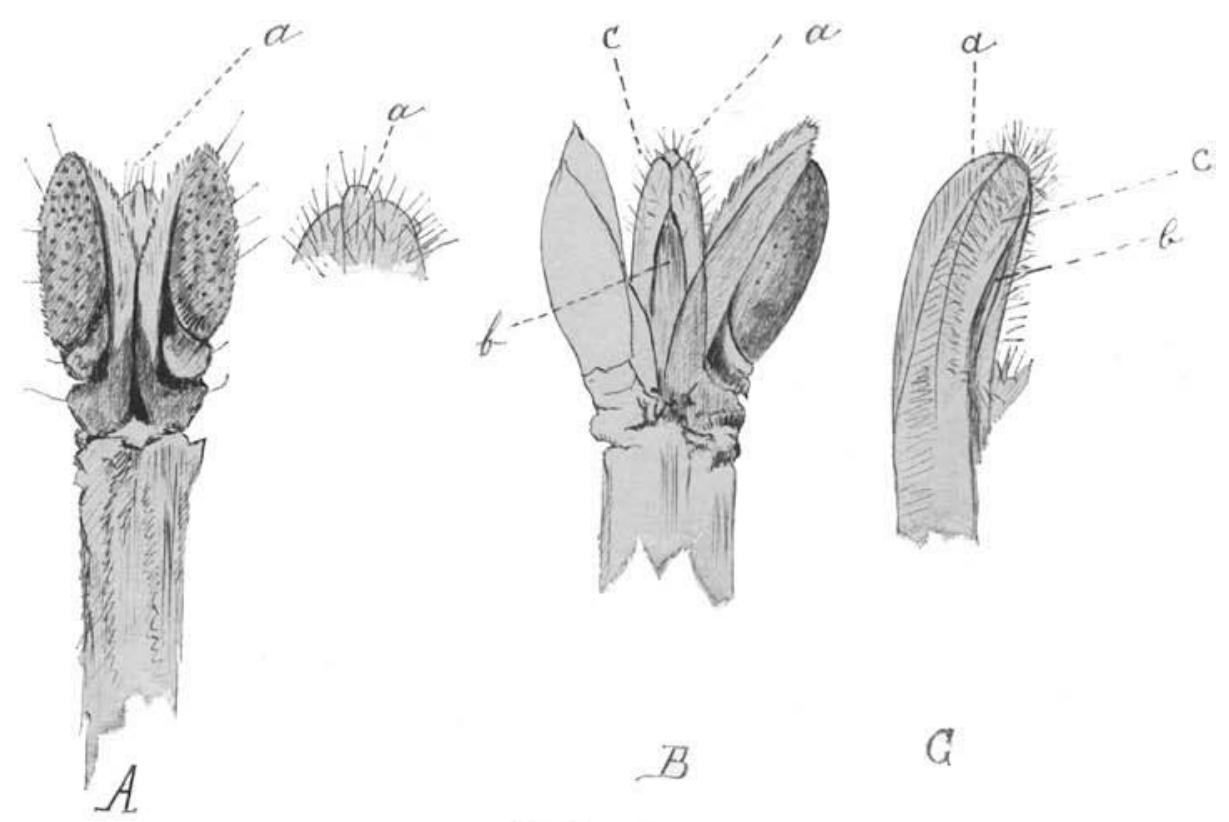

Fit. 5.
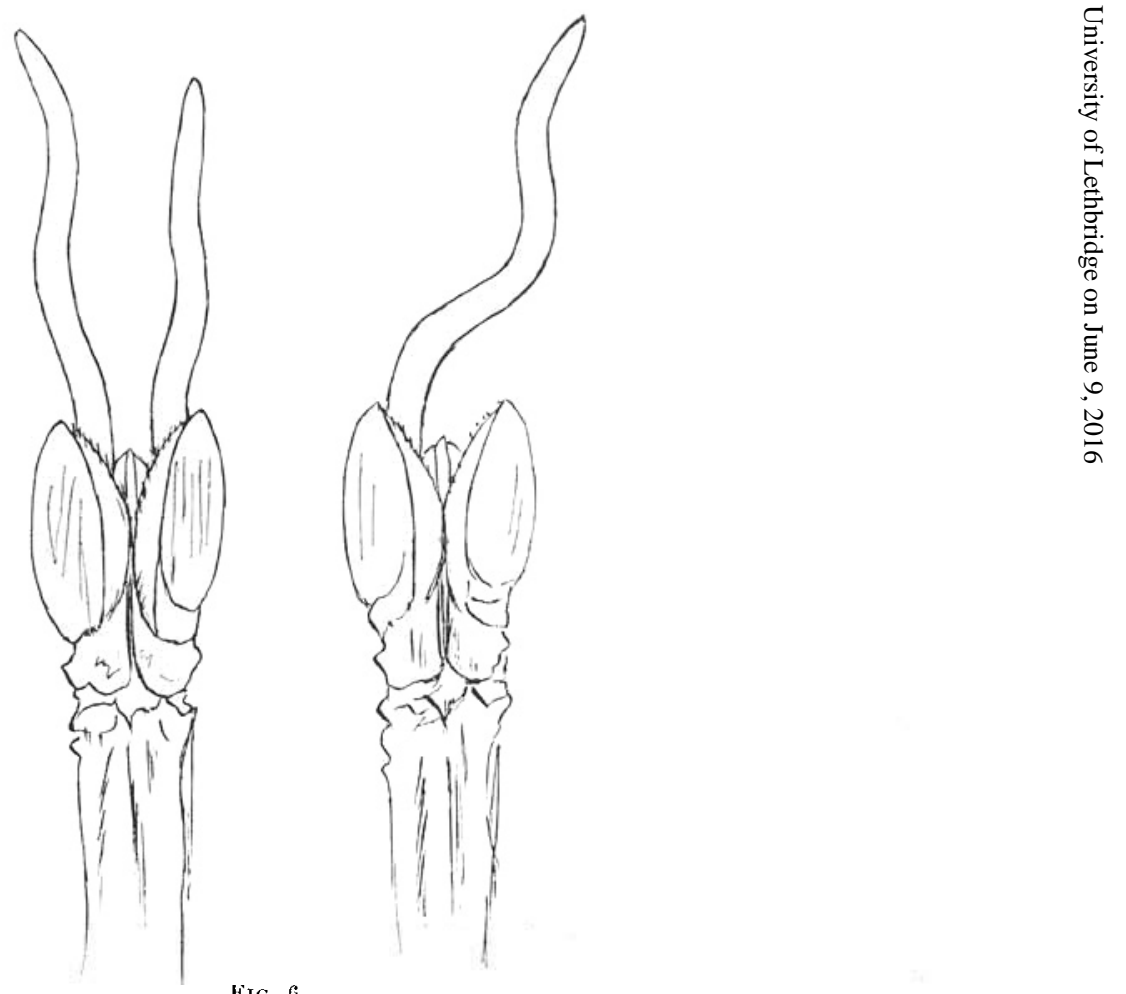

FIG, 6 . 